I. A. Zubareva, The spectrum of the Laplace operator on connected compact simple Lie groups of rank four. II, Mat. Tr., 2019, Volume 22, Number 2, 34-53

DOI: https://doi.org/10.33048/mattrudy.2019.22.203

Use of the all-Russian mathematical portal Math-Net.Ru implies that you have read and agreed to these terms of use

http://www.mathnet.ru/eng/agreement

Download details:

IP : 54.157 .27 .8

April 26, 2023, 18:26:03 


\title{
СПЕКТР ОПЕРАТОРА ЛАПЛАСА НА НЕКОТОРЫХ СВЯЗНЫХ КОМПАКТНЫХ ПРОСТЫХ ГРУППАХ ЛИ РАНГА ЧЕТЫРЕ. II
}

\author{
И. А. Зубарева
}

\begin{abstract}
В работе даются явные вычисления спектра оператора Лапласа для гладких вещественных или комплексных функций на связных компактных простых группах Ли ранга четыре с биинвариантной римановой метрикой, отвечающих системам корней $A_{4}$ и $F_{4}$.
\end{abstract}

Ключевые слова и фразы: оператор Лапласа, спектр, представление группы, форма Киллинга, кривизна Риччи.

\section{Введение}

В работе [2] изучается спектр оператора Лапласа на гладких вещественных функциях, определенных на компактных однородных нормальных римановых многообразиях. Показано, что эту задачу в некотором смысле можно свести к рассмотрению компактных односвязных (связных) простых групп Ли $G$ с биинвариантной (т. е. инвариантной относительно левых и правых сдвигов) римановой метрикой $\nu$. В последнем случае предлагается алгоритм поиска спектра лапласиана через представления алгебр Ли групп Ли $G$.

В работе [6] алгоритм поиска лапласиана из [2] обобщен на случай произвольной связной компактной простой группы Ли $G$, а также вычислены спектры лапласиана всех связных компактных простых групп Ли ранга один и два.

В работе [1] посредством алгоритма поиска лапласиана из [6] проведены явные вычисления спектра лапласиана для всех связных компактных простых групп Ли ранга два и установлена связь полученных формул с теорией чисел и целочисленными тернарными и бинарными квадратичными формами.

Работа [5] посвящена нахождению спектра лапласиана для связных компактных простых групп Ли ранга четыре, отвечающих системам корней $B_{4}, C_{4}$ и $D_{4}$, и установлена связь полученных формул с теорией чисел

\footnotetext{
Работа выполнена при поддержке программы фундаментальных научных исследований СО РАН № I.1.1.4, проект № 0314-2016-0004.

(c) И. А. Зубарева; 2019
} 
и целочисленными квадратичными формами двух, трех и четырех переменных.

В данной работе посредством алгоритма поиска лапласиана из [6] проведены явные вычисления спектра связных компактных простых групп ранга четыре, отвечающих системам корней $A_{4}$ и $F_{4}$.

\section{§1. Предварительные сведения}

Пусть $G$ - связная компактная простая группа Ли с биинвариантной римановой метрикой $\nu$. Множество $\operatorname{Spec}(G, \nu)$ всех собственных значений оператора Лапласа - Бельтрами $\Delta$ на гладких вещественных функциях, определенных на $(G, \nu)$, с учетом кратности собственных значений, т. е. размерности пространств соответствующих собственных функций, называется спектром оператора Лапласа. Спектр группы Ли $(G, \nu)$ можно представить следующим образом:

$$
\operatorname{Spec}(G, \nu)=\left\{0=\lambda_{0}>\lambda_{1} \geq \lambda_{2} \geq \ldots\right\} .
$$

Лапласиан естественным образом обобщается на комплекснозначные функции.

Определение 1. Билинейная (симметричная) форма $k_{\rho}$ на алгебре Ли $\mathfrak{g}$, заданная формулой

$$
k_{\rho}(u, v)=\operatorname{trace}(\rho(u) \rho(v)), \quad u, v \in \mathfrak{g},
$$

называется формой, ассоииированной с представлением $\rho$.

Форма $k_{\text {ad }}$, где $\operatorname{ad}(u)(v):=[u, v]$ - присоединенное представление алгебры Ли g, называется формой Киллинга алгебры Ли g.

Из теоремы 1.2 и следствия 1.2 работы [3] вытекает

Предложение 1. Если $(G, \nu)$ является связной компактной простой $m$-мерной группой Ли с биинвариантной римановой метрикой $\nu$, причем $\nu(e)=-k_{\mathrm{ad}}$, то для присоединенного представления $\mathrm{Ad}$ группы Ли $G$

$$
\lambda_{\mathrm{Ad}}=-1, \quad \operatorname{dim} \mathrm{Ad}=d_{\mathrm{Ad}}=m,
$$

где $\lambda_{\mathrm{Ad}}-$ собственное значение оператора Лапласа, отвечающее присоединенному представлению Ad.

Опуская детали, сразу приведем алгоритм вычисления спектров лапласианов всех групп Ли $(G, \nu)$ с фиксированной алгеброй Ли, сформулированный в [6, следствие 5], предполагающий использование табл. I-IX из [4], в которых $\rho$ обозначает вектор $\beta$.

Теорема 1. Для вычисления спектров лапласианов всех связных компактных простых групп Ли $G$ с простой алгеброй Ли $\mathfrak{g}$ с системой корней Г и биинвариантной римановой метрикой $\nu$ с условием $\nu(e)=-\gamma k_{\mathrm{ad}}$, где $\gamma>0$, нужно выполнить следующие действия: 
1) вычислить выражение $b=\langle\widetilde{\alpha}+\beta, \widetilde{\alpha}+\beta\rangle-\langle\beta, \beta\rangle$, где $\widetilde{\alpha}-$ старший (максимальный) корень, предполагая, что относительно скалярного произведения $\langle\cdot, \cdot\rangle($ на $\mathfrak{t}(\mathbb{R}))$ векторы $\varepsilon_{i}$ из соответствующей таблицы в [4] взаимно ортогональны и единичны;

2) взять скалярное произведение $(\cdot, \cdot)=\frac{1}{b}\langle\cdot, \cdot\rangle$;

3) найти фундаментальные веса $\bar{\omega}_{1}, \ldots, \bar{\omega}_{l}$ алгебры Ли $\mathfrak{g}$ группы Ли $G$ (если $\mathfrak{g}$ имеет ранг l) по соответствующей таблице из [4];

4) для каждого старшего веса $\Lambda \in \Lambda_{1}^{+}(\Gamma)$, т. е. для каждого

$$
\Lambda=\sum_{i=1}^{l} \Lambda_{i} \bar{\omega}_{i}, \quad \text { где } \Lambda_{i} \in \mathbb{Z}_{+}, \quad i=1, \ldots, l,
$$

найти собственное число $\lambda(\Lambda)$ оператора Лапласа, отвечающее старшему весу $\Lambda$, по формуле

$$
\lambda(\Lambda)=-\frac{1}{\gamma}[\langle\Lambda+\beta, \Lambda+\beta\rangle-\langle\beta, \beta\rangle]
$$

и размерность $d(\Lambda+\beta)$ неприводимого комплексного представления комплексной оболочки алгебры Ли $\mathfrak{g}$ со старшим весом $\Lambda$ по формуле

$$
d(\Lambda+\beta)=\prod_{\alpha \in \Gamma^{+}} \frac{(\Lambda+\beta, \alpha)}{(\beta, \alpha)}
$$

5) для каждой решетки $\Lambda(G)$, удовлетворяющей соотношению

$$
\Lambda_{0}(\Gamma) \subseteq \Lambda(G) \subseteq \Lambda_{1}(\Gamma),
$$

где $\Lambda_{0}(\Gamma)$ и $\Lambda_{1}(\Gamma)$ - решетки, порожденные простыми корнями и фундаментальными весами, найденные в п. 1 и п. 3 соответственно, $G-$ группа Ли с алгеброй Ли $\mathfrak{g}$, соответствующая характеристической решетке $\Lambda(G)$, выполнить следующие три пункта:

6) найти множество старших весов $\Lambda^{+}(G)=\Lambda(G) \cap \Lambda_{1}^{+}(\Gamma)$, задав его через фундаментальные веса $\bar{\omega}_{1}, \ldots, \bar{\omega}_{l}$;

7) для каждого старшего веса $\Lambda \in \Lambda^{+}(G)$ на основании п. 4 найти собственное значение $\lambda(\Lambda)$ и размерность $d(\Lambda+\beta)$ неприводимого комплексного представления, отвечающих весу $\Lambda$;

8) найти кратность каждого собственного значения $\lambda$ по формуле

$$
\sigma(\lambda)=\sum_{\Lambda: \lambda(\Lambda)=\gamma \lambda} \prod_{\alpha \in \Gamma^{+}}\left(\frac{(\Lambda+\beta, \alpha)}{(\beta, \alpha)}\right)^{2},
$$

получив таким образом спектр $\operatorname{Spec}(G, \nu)$ группы Ли $G$, отвечающей характеристической решетке $\Lambda(G)$. 
$B$ итоге получаем все спектры $\operatorname{Spec}(G, \nu)$ групп Ли $G$ с алгеброй Ли $\mathfrak{g}$ и метрикой $\nu$.

Замечание 1. В формулах (4) и (5), применяемых в п. 4 и п. 8 теоремы 1 , вместо $(\cdot, \cdot)$ можно использовать любое пропорциональное ему скалярное произведение, в частности, $\langle\cdot, \cdot\rangle$ из п. 1 теоремы 1.

Основную роль в классификации простых групп с заданной алгеброй Ли играет максимальная фундаментальная группа $\Lambda_{1}(\Gamma) / \Lambda_{0}(\Gamma)$. Справедливо следующее утверждение (см., например, [1]).

Предложение 2. Предположим, что максимальная фундаментальная группа $\Lambda_{1}(\Gamma) / \Lambda_{0}(\Gamma)$ алгебры Ли $\mathfrak{g}$ имеет простой порядок. Тогда семейство неизоморфных связных компактных групп Ли с алгеброй Ли $\mathfrak{g}$ состоит из двух групп: односвязной группы Ли $G_{1}$ с центром $\Lambda_{1}(\Gamma) / \Lambda_{0}(\Gamma)$ и решеткой весов $\Lambda_{1}(\Gamma)$, совпадающей с решеткой весов алгебры Ли $\Lambda(\mathfrak{g})$, и группы Ли $G_{0}$ без центра с фундаментальной группой $\Lambda_{1}(\Gamma) / \Lambda_{0}(\Gamma)$ и решеткой весов $\Lambda_{0}(\Gamma)$.

Из описания всех неприводимых систем корней в [4, табл. I-IX] видим, что различных неприводимых систем ранга четыре всего пять: $A_{4}, B_{4}, C_{4}$, $D_{4}, F_{4}$.

Таблица 1. Связные компактные простые группы Ли ранга четыре, отвечающие системам корней $A_{4}, F_{4}$

\begin{tabular}{|c|c|c|c|c|c|}
\hline Группа Ли $G$ & $\Gamma$ & $\Lambda(G)$ & $\pi_{1}(G)$ & $C(G)$ & $\operatorname{dim} G$ \\
\hline $\mathrm{SU}(5)$ & $A_{4}$ & $\Lambda_{1}$ & 0 & $\mathbb{Z}_{5}$ & 24 \\
\hline $\mathrm{PSU}(5):=\mathrm{SU}(5) / C(\mathrm{SU}(5))$ & $A_{4}$ & $\Lambda_{0}$ & $\mathbb{Z}_{5}$ & 0 & 24 \\
\hline$F_{4}$ & $F_{4}$ & $\Lambda_{1}$ & 0 & 0 & 52 \\
\hline
\end{tabular}

Используя теорему 1 и табл. 1, в следующих разделах найдем спектры лапласиана всех связных компактных простых групп Ли ранга четыре, отвечающих системам корней $A_{4}$ и $F_{4}$.

\section{§2. Вычисление спектра лапласиана групп $\mathrm{SU}(5)$ и $\mathrm{PSU}(5)$}

Из табл. 1 следует, что рассматриваемым группам Ли отвечает система корней $A_{4}$. Применяем табл. I из [4].

Простые корни:

$$
\alpha_{i}=\varepsilon_{i}-\varepsilon_{i+1}, \quad i=1,2,3,4
$$

максимальный корень $\widetilde{\alpha}=\varepsilon_{1}-\varepsilon_{5}$. 
Положительные корни:

$$
\varepsilon_{i}-\varepsilon_{j}, \quad 1 \leq i<j \leq 5
$$

Сумма положительных корней равна $2 \beta=4 \varepsilon_{1}+2 \varepsilon_{2}-2 \varepsilon_{4}-4 \varepsilon_{5}$, откуда

$$
\beta=2 \varepsilon_{1}+\varepsilon_{2}-\varepsilon_{4}-2 \varepsilon_{5}, \quad \widetilde{\alpha}+\beta=3 \varepsilon_{1}+\varepsilon_{2}-\varepsilon_{4}-3 \varepsilon_{5} .
$$

Действуем согласно алгоритму, приведенному в теореме 1.

1) $b=\langle\widetilde{\alpha}+\beta, \widetilde{\alpha}+\beta\rangle-\langle\beta, \beta\rangle=20-10=10$.

2) $(\cdot, \cdot)=\frac{1}{10}\langle\cdot, \cdot\rangle$.

3) Фундаментальные веса имеют вид

$$
\begin{array}{ll}
\bar{\omega}_{1}=\frac{4 \varepsilon_{1}-\varepsilon_{2}-\varepsilon_{3}-\varepsilon_{4}-\varepsilon_{5}}{5}, & \bar{\omega}_{2}=\frac{3 \varepsilon_{1}+3 \varepsilon_{2}-2 \varepsilon_{3}-2 \varepsilon_{4}-2 \varepsilon_{5}}{5}, \\
\bar{\omega}_{3}=\frac{2 \varepsilon_{1}+2 \varepsilon_{2}+2 \varepsilon_{3}-3 \varepsilon_{4}-3 \varepsilon_{5}}{5}, & \bar{\omega}_{4}=\frac{\varepsilon_{1}+\varepsilon_{2}+\varepsilon_{3}+\varepsilon_{4}-4 \varepsilon_{5}}{5} .
\end{array}
$$

Легко видеть, что

$$
\widetilde{\alpha}=\bar{\omega}_{1}+\bar{\omega}_{4}, \quad \beta=\bar{\omega}_{1}+\bar{\omega}_{2}+\bar{\omega}_{3}+\bar{\omega}_{4} .
$$

4) Пусть

$$
\Lambda=\sum_{i=1}^{4} \Lambda_{i} \bar{\omega}_{i}, \quad \text { где } \quad \Lambda_{i} \in \mathbb{Z}, \quad \Lambda_{i} \geq 0, \quad i=1,2,3,4 .
$$

Тогда

$$
\begin{aligned}
\Lambda+\beta=\sum_{i=1}^{4} & \left(\Lambda_{i}+1\right) \bar{\omega}_{i} \\
=\frac{1}{5}[ & \left(4 \nu_{1}+3 \nu_{2}+2 \nu_{3}+\nu_{4}\right) \varepsilon_{1}+\left(-\nu_{1}+3 \nu_{2}+2 \nu_{3}+\nu_{4}\right) \varepsilon_{2} \\
& \quad+\left(-\nu_{1}-2 \nu_{2}+2 \nu_{3}+\nu_{4}\right) \varepsilon_{3}+\left(-\nu_{1}-2 \nu_{2}-3 \nu_{3}+\nu_{4}\right) \varepsilon_{4} \\
& \left.\quad-\left(\nu_{1}+2 \nu_{2}+3 \nu_{3}+4 \nu_{4}\right) \varepsilon_{5}\right]
\end{aligned}
$$

где $\nu_{i}=\Lambda_{i}+1, \nu_{i} \in \mathbb{N}, i=1,2,3,4$,

Найдем собственное значение $\lambda(\Lambda)$, соответствующее старшему весу $\Lambda$, по формуле (3):

$$
\begin{aligned}
\lambda(\Lambda)=-\frac{1}{250 \gamma} & {\left[\left(4 \nu_{1}+3 \nu_{2}+2 \nu_{3}+\nu_{4}\right)^{2}+\left(-\nu_{1}+3 \nu_{2}+2 \nu_{3}+\nu_{4}\right)^{2}\right.} \\
& +\left(-\nu_{1}-2 \nu_{2}+2 \nu_{3}+\nu_{4}\right)^{2}+\left(-\nu_{1}-2 \nu_{2}-3 \nu_{3}+\nu_{4}\right)^{2} \\
& \left.+\left(\nu_{1}+2 \nu_{2}+3 \nu_{3}+4 \nu_{4}\right)^{2}-250\right] .
\end{aligned}
$$


Проводя рутинные вычисления, получаем, что

$$
\begin{aligned}
\lambda(\Lambda)=-\frac{1}{50 \gamma}[ & 4 \nu_{1}^{2}+6 \nu_{1} \nu_{2}+4 \nu_{1} \nu_{3}+2 \nu_{1} \nu_{4}+6 \nu_{2}^{2} \\
& \left.+8 \nu_{2} \nu_{3}+4 \nu_{2} \nu_{4}+6 \nu_{3}^{2}+6 \nu_{3} \nu_{4}+4 \nu_{4}^{2}-50\right] .
\end{aligned}
$$

Вычислим размерность $d(\Lambda+\beta)$ представления $\rho(\Lambda)$, отвечающего старшему весу $\Lambda$, по формуле (4):

$$
\begin{aligned}
& d(\Lambda+\beta)=\frac{\left(\Lambda+\beta, \varepsilon_{1}-\varepsilon_{2}\right)}{\left(\beta, \varepsilon_{1}-\varepsilon_{2}\right)} \cdot \frac{\left(\Lambda+\beta, \varepsilon_{1}-\varepsilon_{3}\right)}{\left(\beta, \varepsilon_{1}-\varepsilon_{3}\right)} \cdot \frac{\left(\Lambda+\beta, \varepsilon_{1}-\varepsilon_{4}\right)}{\left(\beta, \varepsilon_{1}-\varepsilon_{4}\right)} \cdot \frac{\left(\Lambda+\beta, \varepsilon_{1}-\varepsilon_{5}\right)}{\left(\beta, \varepsilon_{1}-\varepsilon_{5}\right)} \\
& \times \frac{\left(\Lambda+\beta, \varepsilon_{2}-\varepsilon_{3}\right)}{\left(\beta, \varepsilon_{2}-\varepsilon_{3}\right)} \cdot \frac{\left(\Lambda+\beta, \varepsilon_{2}-\varepsilon_{4}\right)}{\left(\beta, \varepsilon_{2}-\varepsilon_{4}\right)} \cdot \frac{\left(\Lambda+\beta, \varepsilon_{2}-\varepsilon_{5}\right)}{\left(\beta, \varepsilon_{2}-\varepsilon_{5}\right)} \\
& \times \frac{\left(\Lambda+\beta, \varepsilon_{3}-\varepsilon_{4}\right)}{\left(\beta, \varepsilon_{3}-\varepsilon_{4}\right)} \cdot \frac{\left(\Lambda+\beta, \varepsilon_{3}-\varepsilon_{5}\right)}{\left(\beta, \varepsilon_{3}-\varepsilon_{5}\right)} \cdot \frac{\left(\Lambda+\beta, \varepsilon_{4}-\varepsilon_{5}\right)}{\left(\beta, \varepsilon_{4}-\varepsilon_{5}\right)} .
\end{aligned}
$$

На основании (6) и (7) имеем

$$
\begin{array}{cl}
\frac{\left(\Lambda+\beta, \varepsilon_{1}-\varepsilon_{2}\right)}{\left(\beta, \varepsilon_{1}-\varepsilon_{2}\right)}=\nu_{1}, & \frac{\left(\Lambda+\beta, \varepsilon_{1}-\varepsilon_{3}\right)}{\left(\beta, \varepsilon_{1}-\varepsilon_{3}\right)}=\frac{\nu_{1}+\nu_{2}}{2}, \\
\frac{\left(\Lambda+\beta, \varepsilon_{1}-\varepsilon_{4}\right)}{\left(\beta, \varepsilon_{1}-\varepsilon_{4}\right)}=\frac{\nu_{1}+\nu_{2}+\nu_{3}}{3}, & \frac{\left(\Lambda+\beta, \varepsilon_{1}-\varepsilon_{5}\right)}{\left(\beta, \varepsilon_{1}-\varepsilon_{5}\right)}=\frac{\nu_{1}+\nu_{2}+\nu_{3}+\nu_{4}}{4}, \\
\frac{\left(\Lambda+\beta, \varepsilon_{2}-\varepsilon_{3}\right)}{\left(\beta, \varepsilon_{2}-\varepsilon_{3}\right)}=\nu_{2}, & \frac{\left(\Lambda+\beta, \varepsilon_{2}-\varepsilon_{4}\right)}{\left(\beta, \varepsilon_{2}-\varepsilon_{4}\right)}=\frac{\nu_{2}+\nu_{3}}{2}, \\
\frac{\left(\Lambda+\beta, \varepsilon_{2}-\varepsilon_{5}\right)}{\left(\beta, \varepsilon_{2}-\varepsilon_{5}\right)}=\frac{\nu_{2}+\nu_{3}+\nu_{4}}{3}, \quad \frac{\left(\Lambda+\beta, \varepsilon_{3}-\varepsilon_{4}\right)}{\left(\beta, \varepsilon_{3}-\varepsilon_{4}\right)}=\nu_{3}, \\
\frac{\left(\Lambda+\beta, \varepsilon_{3}-\varepsilon_{5}\right)}{\left(\beta, \varepsilon_{3}-\varepsilon_{5}\right)}=\frac{\nu_{3}+\nu_{4}}{2}, \quad \frac{\left(\Lambda+\beta, \varepsilon_{4}-\varepsilon_{5}\right)}{\left(\beta, \varepsilon_{4}-\varepsilon_{5}\right)}=\nu_{4} .
\end{array}
$$

Следовательно,

$$
\begin{aligned}
d(\Lambda+\beta)= & \frac{1}{288} \nu_{1} \nu_{2} \nu_{3} \nu_{4}\left(\nu_{1}+\nu_{2}\right)\left(\nu_{2}+\nu_{3}\right)\left(\nu_{3}+\nu_{4}\right)\left(\nu_{1}+\nu_{2}+\nu_{3}\right) \\
& \times\left(\nu_{2}+\nu_{3}+\nu_{4}\right)\left(\nu_{1}+\nu_{2}+\nu_{3}+\nu_{4}\right) .
\end{aligned}
$$

Замечание 2. Из (8) и (9) вытекает, что

$$
\lambda(\Lambda)=0 \Leftrightarrow \Lambda=0 \Leftrightarrow \nu_{1}=\nu_{2}=\nu_{3}=\nu_{4},
$$

и, следовательно, $\sigma\left(\lambda_{0}\right)=d^{2}(0+\beta)=1$, что соответствует формуле $(1)$. Кроме того, на основании предложения 1 старшим весом присоединенного представления $\mathrm{Ad}$ является максимальный корень $\widetilde{\alpha}=\bar{\omega}_{1}+\bar{\omega}_{4}$, для 
которого $\nu_{1}=\nu_{4}=2$ и $\nu_{2}=\nu_{3}=1$. Поэтому собственное значение и размерность представления Ad можно вычислить следующим образом:

$$
\begin{gathered}
\lambda_{\mathrm{Ad}}=\lambda(\widetilde{\alpha})=-\frac{1}{50 \gamma}(16+12+8+8+6+8+8+6+12+16-50)=-\frac{1}{\gamma}, \\
\operatorname{dim~Ad}=d(\widetilde{\alpha}+\beta)=\frac{2 \cdot 2 \cdot 3 \cdot 2 \cdot 3 \cdot 4 \cdot 4 \cdot 6}{288}=24,
\end{gathered}
$$

что соответствует формуле (2) при $\gamma=1$ с учетом равенства $\operatorname{dim} \mathrm{SU}(5)=$ 24 из табл. 1.

5) Указанные выше простые корни и фундаментальные веса алгебры Ли $\mathfrak{s u}(5)$ задают решетки $\Lambda_{0}\left(A_{4}\right)$ и $\Lambda_{1}\left(A_{4}\right)$ соответственно следующим образом:

$$
\begin{aligned}
& \Lambda_{0}\left(A_{4}\right)=\left\{\sum_{i=1}^{4} \Psi_{i} \alpha_{i} \mid \Psi_{i} \in \mathbb{Z}, \quad i=1,2,3,4\right\}, \\
& \Lambda_{1}\left(A_{4}\right)=\left\{\sum_{i=1}^{4} \Lambda_{i} \bar{\omega}_{i} \mid \Lambda_{i} \in \mathbb{Z}, \quad i=1,2,3,4\right\} .
\end{aligned}
$$

После выражения корней через фундаментальные веса $\alpha_{1}=2 \bar{\omega}_{1}-\bar{\omega}_{2}, \quad \alpha_{2}=-\bar{\omega}_{1}+2 \bar{\omega}_{2}-\bar{\omega}_{3}, \quad \alpha_{3}=-\bar{\omega}_{2}+2 \bar{\omega}_{3}-\bar{\omega}_{4}, \quad \alpha_{4}=-\bar{\omega}_{3}+2 \bar{\omega}_{4}$ и замены переменных:

$$
\left\{\begin{array} { l } 
{ \Psi _ { 1 } = 4 \Omega _ { 1 } - \Omega _ { 2 } - 2 \Omega _ { 3 } - 3 \Omega _ { 4 } , } \\
{ \Psi _ { 2 } = 3 \Omega _ { 1 } - \Omega _ { 3 } - 2 \Omega _ { 4 } , } \\
{ \Psi _ { 3 } = 2 \Omega _ { 1 } - \Omega _ { 4 } , } \\
{ \Psi _ { 4 } = \Omega _ { 1 } , }
\end{array} \Leftrightarrow \left\{\begin{array}{l}
\Omega_{1}=\Psi_{4}, \\
\Omega_{2}=-\Psi_{1}+2 \Psi_{2}-\Psi_{3}, \\
\Omega_{3}=-\Psi_{2}+2 \Psi_{3}-\Psi_{4}, \\
\Omega_{4}=-\Psi_{3}+2 \Psi_{4}
\end{array}\right.\right.
$$

решетка $\Lambda_{0}\left(A_{4}\right)$ приобретает следующий вид:

$$
\begin{aligned}
\Lambda_{0}\left(A_{4}\right)=\{ & 5 \Omega_{1} \bar{\omega}_{1}+\Omega_{2}\left(\bar{\omega}_{2}-2 \bar{\omega}_{1}\right)+\Omega_{3}\left(\bar{\omega}_{3}-3 \bar{\omega}_{1}\right) \\
& \left.+\Omega_{4}\left(\bar{\omega}_{4}-4 \bar{\omega}_{1}\right) \mid \Omega_{i} \in \mathbb{Z}, \quad i=1,2,3,4\right\} .
\end{aligned}
$$

Определим решетку $\Lambda_{1}\left(A_{4}\right)$ в базисе $\left\{\bar{\omega}_{1}, \bar{\omega}_{2}-2 \bar{\omega}_{1}, \bar{\omega}_{3}-3 \bar{\omega}_{1}, \bar{\omega}_{4}-4 \bar{\omega}_{1}\right\}$ посредством замены переменных $\Lambda_{1}=\Omega_{1}-2 \Omega_{2}-3 \Omega_{3}-4 \Omega_{4}, \Lambda_{2}=\Omega_{2}$, $\Lambda_{3}=\Omega_{3}, \Lambda_{4}=\Omega_{4}$ :

$$
\begin{aligned}
\Lambda_{1}\left(A_{4}\right)=\{ & \Omega_{1} \bar{\omega}_{1}+\Omega_{2}\left(\bar{\omega}_{2}-2 \bar{\omega}_{1}\right)+\Omega_{3}\left(\bar{\omega}_{3}-3 \bar{\omega}_{1}\right) \\
& \left.+\Omega_{4}\left(\bar{\omega}_{4}-4 \bar{\omega}_{1}\right) \mid \Omega_{i} \in \mathbb{Z}, \quad i=1,2,3,4\right\} .
\end{aligned}
$$

Из (10) и (11) следует, что

$$
\Lambda_{0}\left(A_{4}\right) \subset \Lambda_{1}\left(A_{4}\right) \text { и } \Lambda_{1}\left(A_{4}\right) / \Lambda_{0}\left(A_{4}\right) \cong \mathbb{Z}_{5},
$$


т. е. $\Lambda_{1}\left(A_{4}\right) / \Lambda_{0}\left(A_{4}\right)$ имеет простой порядок. Отсюда на основании следствия 2 заключаем, что других решеток нет. Группы Ли, отвечающие данным характеристическим решеткам, приведены в табл. 1.

а) Приведем формулы, задающие спектр лапласиана группы Ли SU(5).

6а) Множество старших весов группы Ли SU(5) получаем из формулы (10):

$$
\Lambda^{+}(\mathrm{SU}(5))=\left\{\sum_{i=1}^{4} \Lambda_{i} \bar{\omega}_{i} \mid \Lambda_{i} \in \mathbb{Z}, \quad \Lambda_{i} \geq 0, \quad i=1,2,3,4\right\} .
$$

7а) Пусть

$$
\Lambda=\sum_{i=1}^{4} \Lambda_{i} \bar{\omega}_{i} \in \Lambda^{+}(\mathrm{SU}(5)) .
$$

Тогда на основании п. 4 собственное число $\lambda(\Lambda)$ и размерность $d(\Lambda+\beta)$ вычисляются по формулам (8) и (9) соответственно, где $\nu_{i}=\Lambda_{i}+1, \nu_{i} \in \mathbb{N}$, $i=1,2,3,4$.

8a) Применяя формулу (5) и результаты предыдущего пункта, получаем кратность собственного значения $\lambda$ :

$$
\begin{aligned}
\sigma(\Lambda)= & \frac{1}{288^{2}} \sum_{\Xi_{1}} \nu_{1}^{2} \nu_{2}^{2} \nu_{3}^{2} \nu_{4}^{2}\left(\nu_{1}+\nu_{2}\right)^{2}\left(\nu_{2}+\nu_{3}\right)^{2}\left(\nu_{3}+\nu_{4}\right)^{2}\left(\nu_{1}+\nu_{2}+\nu_{3}\right)^{2} \\
& \times\left(\nu_{2}+\nu_{3}+\nu_{4}\right)^{2}\left(\nu_{1}+\nu_{2}+\nu_{3}+\nu_{4}\right)^{2}
\end{aligned}
$$

где

$$
\begin{gathered}
\Xi_{1}=\{4 \\
4 \nu_{1}^{2}+6 \nu_{1} \nu_{2}+4 \nu_{1} \nu_{3}+2 \nu_{1} \nu_{4}+6 \nu_{2}^{2}+8 \nu_{2} \nu_{3}+4 \nu_{2} \nu_{4}+6 \nu_{3}^{2} \\
\left.+6 \nu_{3} \nu_{4}+4 \nu_{4}^{2}=50-50 \gamma \lambda \mid \nu_{i} \in \mathbb{N}\right\} .
\end{gathered}
$$

Наименьшее по модулю ненулевое значение лапласиана равно $-\frac{12}{25 \gamma}$ и соответствует неприводимым представлениям группы Ли $\mathrm{SU}(5)$ со старшими весами $\bar{\omega}_{1}$ и $\bar{\omega}_{4}$. Размерности этих представлений равны 5 . Следовательно, кратность собственного значения $-\frac{12}{25 \gamma}$ равна $5^{2}+5^{2}=50$.

b) Ниже приводим формулы, которые задают спектр лапласиана группы Ли PSU(5).

6b) Множество старших весов группы Ли PSU(5) получаем из формулы (11):

$$
\begin{aligned}
\Lambda^{+}(\operatorname{PSU}(5))=\{ & \left(5 \Omega_{1}-2 \Omega_{2}-3 \Omega_{3}-4 \Omega_{4}\right) \bar{\omega}_{1}+\Omega_{2} \bar{\omega}_{2}+\Omega_{3} \bar{\omega}_{3} \\
& +\Omega_{4} \bar{\omega}_{4} \mid \Omega_{i} \in \mathbb{Z}, \quad i=1,2,3,4 \\
& \left.5 \Omega_{1}-2 \Omega_{2}-3 \Omega_{3}-4 \Omega_{4} \geq 0, \Omega_{2} \geq 0, \Omega_{3} \geq 0, \Omega_{4} \geq 0\right\} .
\end{aligned}
$$

7b) Пусть

$$
\Lambda=\Omega_{1} \bar{\omega}_{1}+\Omega_{2} \bar{\omega}_{2}+\Omega_{3} \bar{\omega}_{3}+2 \Omega_{4} \bar{\omega}_{4} \in \Lambda^{+}(\operatorname{PSU}(5)) .
$$


Тогда на основании п. 4 собственное число $\lambda(\Lambda)$ и размерность $d(\Lambda+\beta)$ вычисляются по формулам (8) и (9) соответственно, где

$$
\begin{gathered}
\nu_{1}=5 \Omega_{1}-2 \Omega_{2}-3 \Omega_{3}-4 \Omega_{4}+1, \quad \nu_{2}=\Omega_{2}+1, \quad \nu_{3}=\Omega_{3}+1, \quad \nu_{4}=\Omega_{4}+1, \\
\nu_{i} \in \mathbb{N}, \quad i=1,2,3,4 ; \quad \nu_{1}+2 \nu_{2}+3 \nu_{3}+4 \nu_{4} \equiv 0 .
\end{gathered}
$$

8b) Применяя формулу (5) и результаты предыдущего пункта, получаем кратность собственного значения $\lambda$ :

$$
\begin{gathered}
\sigma(\Lambda)=\frac{1}{288^{2}} \sum_{\Xi_{2}} \nu_{1}^{2} \nu_{2}^{2} \nu_{3}^{2} \nu_{4}^{2}\left(\nu_{1}+\nu_{2}\right)^{2}\left(\nu_{2}+\nu_{3}\right)^{2}\left(\nu_{3}+\nu_{4}\right)^{2}\left(\nu_{1}+\nu_{2}+\nu_{3}\right)^{2} \\
\times\left(\nu_{2}+\nu_{3}+\nu_{4}\right)^{2}\left(\nu_{1}+\nu_{2}+\nu_{3}+\nu_{4}\right)^{2}
\end{gathered}
$$

где

$$
\begin{gathered}
\Xi_{2}=\left\{4 \nu_{1}^{2}+6 \nu_{1} \nu_{2}+4 \nu_{1} \nu_{3}+2 \nu_{1} \nu_{4}+6 \nu_{2}^{2}+8 \nu_{2} \nu_{3}+4 \nu_{2} \nu_{4}+6 \nu_{3}^{2}+6 \nu_{3} \nu_{4}+4 \nu_{4}^{2}\right. \\
\left.=50-50 \gamma \lambda \mid \nu_{1}+2 \nu_{2}+3 \nu_{3}+4 \nu_{4} \equiv 0(\bmod 5), \nu_{i} \in \mathbb{N}\right\} .
\end{gathered}
$$

Наименьшее по модулю ненулевое собственное значение лапласиана равно -1 и соответствует неприводимому комплексному представлению группы Ли PSU(5) со старшим весом $\bar{\omega}_{1}+\bar{\omega}_{4}$. Размерность этого представления равна 24. Следовательно, кратность собственного значения $-\frac{23}{25 \gamma}$ равна $24^{2}=576$.

\section{§3. Вычисление спектра лапласиана для группы $\boldsymbol{F}_{4}$}

Группе $F_{4}$ с алгеброй Ли $\mathfrak{f}_{4}$ соответствует система корней $F_{4}$. Применим табл. VIII из [4].

Простые корни:

$$
\alpha_{1}=\varepsilon_{2}-\varepsilon_{3}, \quad \alpha_{2}=\varepsilon_{3}-\varepsilon_{4}, \quad \alpha_{3}=\varepsilon_{4}, \quad \alpha_{4}=\frac{1}{2}\left(\varepsilon_{1}-\varepsilon_{2}-\varepsilon_{3}-\varepsilon_{4}\right) ;
$$

максимальный корень $\widetilde{\alpha}=\varepsilon_{1}+\varepsilon_{2}$.

Положительные корни:

$$
\varepsilon_{i}, \quad i=1,2,3,4 ; \quad \varepsilon_{i} \pm \varepsilon_{j}, \quad 1 \leq i<j \leq 4 ; \quad \frac{1}{2}\left(\varepsilon_{1} \pm \varepsilon_{2} \pm \varepsilon_{3} \pm \varepsilon_{4}\right) .
$$

Сумма положительных корней равна $2 \beta=11 \varepsilon_{1}+5 \varepsilon_{2}+3 \varepsilon_{3}+\varepsilon_{4}$, откуда

$$
\beta=\frac{11 \varepsilon_{1}+5 \varepsilon_{2}+3 \varepsilon_{3}+\varepsilon_{4}}{2}, \quad \widetilde{\alpha}+\beta=\frac{13 \varepsilon_{1}+7 \varepsilon_{2}+3 \varepsilon_{3}+\varepsilon_{4}}{2} .
$$

Действуем согласно алгоритму, приведенному в теореме 1. 
1) $b=\langle\widetilde{\alpha}+\beta, \widetilde{\alpha}+\beta\rangle-\langle\beta, \beta\rangle=57-39=18$.

2) $(\cdot, \cdot)=\frac{1}{18}\langle\cdot, \cdot\rangle$.

3) Фундаментальные веса имеют вид

$$
\bar{\omega}_{1}=\varepsilon_{1}+\varepsilon_{2}, \quad \bar{\omega}_{2}=2 \varepsilon_{1}+\varepsilon_{2}+\varepsilon_{3}, \quad \bar{\omega}_{3}=\frac{3 \varepsilon_{1}+\varepsilon_{2}+\varepsilon_{3}+\varepsilon_{4}}{2}, \quad \bar{\omega}_{4}=\varepsilon_{1} .
$$

Легко видеть, что

$$
\widetilde{\alpha}=\bar{\omega}_{1}, \quad \beta=\bar{\omega}_{1}+\bar{\omega}_{2}+\bar{\omega}_{3}+\bar{\omega}_{4}
$$

4) Пусть

Тогда

$$
\Lambda=\sum_{i=1}^{4} \Lambda_{i} \bar{\omega}_{i}, \quad \text { где } \Lambda_{i} \in \mathbb{Z}, \quad \Lambda_{i} \geq 0, \quad i=1,2,3,4 .
$$

$$
\begin{aligned}
\Lambda+\beta= & \sum_{i=1}^{4}\left(\Lambda_{i}+1\right) \bar{\omega}_{i}=\sum_{i=1}^{4} \nu_{i} \bar{\omega}_{i} \\
= & \frac{1}{2}\left[\left(2 \nu_{1}+4 \nu_{2}+3 \nu_{3}+2 \nu_{4}\right) \varepsilon_{1}+\left(2 \nu_{1}+2 \nu_{2}+\nu_{3}\right) \varepsilon_{2}\right. \\
& \left.\quad+\left(2 \nu_{2}+\nu_{3}\right) \varepsilon_{3}+\nu_{3} \varepsilon_{4}\right]
\end{aligned}
$$

где $\nu_{i}=\Lambda_{i}+1, \nu_{i} \in \mathbb{N}, i=1,2,3,4$.

Найдем собственное значение $\lambda(\Lambda)$, соответствующее старшему весу $\Lambda$, по формуле (3):

$$
\begin{gathered}
\lambda(\Lambda)=-\frac{1}{18 \gamma}[\langle\Lambda+\beta, \Lambda+\beta\rangle-\langle\beta, \beta\rangle] \\
=-\frac{1}{72 \gamma}\left[\left(2 \nu_{1}+4 \nu_{2}+3 \nu_{3}+2 \nu_{4}\right)^{2}+\left(2 \nu_{1}+2 \nu_{2}+\nu_{3}\right)^{2}\right. \\
\left.+\left(2 \nu_{2}+\nu_{3}\right)^{2}+\nu_{3}^{2}-156\right]
\end{gathered}
$$

Теперь вычислим размерность $d(\Lambda+\beta)$ представления $\rho(\Lambda)$, отвечающего старшему весу $\Lambda$, по формуле (4):

$$
\begin{aligned}
& d(\Lambda+\beta)=\frac{\left(\Lambda+\beta, \varepsilon_{1}\right)}{\left(\beta, \varepsilon_{1}\right)} \cdot \frac{\left(\Lambda+\beta, \varepsilon_{2}\right)}{\left(\beta, \varepsilon_{2}\right)} \cdot \frac{\left(\Lambda+\beta, \varepsilon_{3}\right)}{\left(\beta, \varepsilon_{3}\right)} \cdot \frac{\left(\Lambda+\beta, \varepsilon_{4}\right)}{\left(\beta, \varepsilon_{4}\right)} \\
& \times \frac{\left(\Lambda+\beta, \varepsilon_{1}-\varepsilon_{2}\right)}{\left(\beta, \varepsilon_{1}-\varepsilon_{2}\right)} \cdot \frac{\left(\Lambda+\beta, \varepsilon_{1}-\varepsilon_{3}\right)}{\left(\beta, \varepsilon_{1}-\varepsilon_{3}\right)} \cdot \frac{\left(\Lambda+\beta, \varepsilon_{1}-\varepsilon_{4}\right)}{\left(\beta, \varepsilon_{1}-\varepsilon_{4}\right)} \\
& \times \frac{\left(\Lambda+\beta, \varepsilon_{2}-\varepsilon_{3}\right)}{\left(\beta, \varepsilon_{2}-\varepsilon_{3}\right)} \cdot \frac{\left(\Lambda+\beta, \varepsilon_{2}-\varepsilon_{4}\right)}{\left(\beta, \varepsilon_{2}-\varepsilon_{4}\right)} \cdot \frac{\left(\Lambda+\beta, \varepsilon_{3}-\varepsilon_{4}\right)}{\left(\beta, \varepsilon_{3}-\varepsilon_{4}\right)} \\
& \times \frac{\left(\Lambda+\beta, \varepsilon_{1}+\varepsilon_{2}\right)}{\left(\beta, \varepsilon_{1}+\varepsilon_{2}\right)} \cdot \frac{\left(\Lambda+\beta, \varepsilon_{1}+\varepsilon_{3}\right)}{\left(\beta, \varepsilon_{1}+\varepsilon_{3}\right)} \cdot \frac{\left(\Lambda+\beta, \varepsilon_{1}+\varepsilon_{4}\right)}{\left(\beta, \varepsilon_{1}+\varepsilon_{4}\right)}
\end{aligned}
$$




$$
\begin{aligned}
& \times \frac{\left(\Lambda+\beta, \varepsilon_{2}+\varepsilon_{3}\right)}{\left(\beta, \varepsilon_{2}+\varepsilon_{3}\right)} \cdot \frac{\left(\Lambda+\beta, \varepsilon_{2}+\varepsilon_{4}\right)}{\left(\beta, \varepsilon_{2}+\varepsilon_{4}\right)} \cdot \frac{\left(\Lambda+\beta, \varepsilon_{3}+\varepsilon_{4}\right)}{\left(\beta, \varepsilon_{3}+\varepsilon_{4}\right)} \\
& \times \frac{\left(\Lambda+\beta, \frac{1}{2}\left(\varepsilon_{1}+\varepsilon_{2}+\varepsilon_{3}+\varepsilon_{4}\right)\right)}{\left(\beta, \frac{1}{2}\left(\varepsilon_{1}+\varepsilon_{2}+\varepsilon_{3}+\varepsilon_{4}\right)\right)} \cdot \frac{\left(\Lambda+\beta, \frac{1}{2}\left(\varepsilon_{1}+\varepsilon_{2}+\varepsilon_{3}-\varepsilon_{4}\right)\right)}{\left(\beta, \frac{1}{2}\left(\varepsilon_{1}+\varepsilon_{2}+\varepsilon_{3}-\varepsilon_{4}\right)\right)} \\
& \times \frac{\left(\Lambda+\beta, \frac{1}{2}\left(\varepsilon_{1}+\varepsilon_{2}-\varepsilon_{3}+\varepsilon_{4}\right)\right)}{\left(\beta, \frac{1}{2}\left(\varepsilon_{1}+\varepsilon_{2}-\varepsilon_{3}+\varepsilon_{4}\right)\right)} \cdot \frac{\left(\Lambda+\beta, \frac{1}{2}\left(\varepsilon_{1}+\varepsilon_{2}-\varepsilon_{3}-\varepsilon_{4}\right)\right)}{\left(\beta, \frac{1}{2}\left(\varepsilon_{1}+\varepsilon_{2}-\varepsilon_{3}-\varepsilon_{4}\right)\right)} \\
& \times \frac{\left(\Lambda+\beta, \frac{1}{2}\left(\varepsilon_{1}-\varepsilon_{2}+\varepsilon_{3}+\varepsilon_{4}\right)\right)}{\left(\beta, \frac{1}{2}\left(\varepsilon_{1}-\epsilon_{2}+\varepsilon_{3}+\varepsilon_{4}\right)\right)} \cdot \frac{\left(\Lambda+\beta, \frac{1}{2}\left(\varepsilon_{1}-\varepsilon_{2}-\varepsilon_{3}+\varepsilon_{4}\right)\right)}{\left(\beta, \frac{1}{2}\left(\varepsilon_{1}-\varepsilon_{2}-\varepsilon_{3}+\varepsilon_{4}\right)\right)} \\
& \times \frac{\left(\Lambda+\beta, \frac{1}{2}\left(\varepsilon_{1}-\varepsilon_{2}+\varepsilon_{3}-\varepsilon_{4}\right)\right)}{\left(\beta, \frac{1}{2}\left(\varepsilon_{1}-\varepsilon_{2}+\varepsilon_{3}-\varepsilon_{4}\right)\right)} \cdot \frac{\left(\Lambda+\beta, \frac{1}{2}\left(\varepsilon_{1}-\varepsilon_{2}-\varepsilon_{3}-\varepsilon_{4}\right)\right)}{\left(\beta, \frac{1}{2}\left(\varepsilon_{1}-\varepsilon_{2}-\varepsilon_{3}-\varepsilon_{4}\right)\right)} .
\end{aligned}
$$

На основании (15) и (16) имеем

$$
\begin{aligned}
& \frac{\left(\Lambda+\beta, \varepsilon_{1}\right)}{\left(\beta, \varepsilon_{1}\right)}=\frac{2 \nu_{1}+4 \nu_{2}+3 \nu_{3}+2 \nu_{4}}{11}, \quad \frac{\left(\Lambda+\beta, \varepsilon_{2}\right)}{\left(\beta, \varepsilon_{2}\right)}=\frac{2 \nu_{1}+2 \nu_{2}+\nu_{3}}{5}, \\
& \frac{\left(\Lambda+\beta, \varepsilon_{3}\right)}{\left(\beta, \varepsilon_{3}\right)}=\frac{2 \nu_{2}+\nu_{3}}{3}, \quad \frac{\left(\Lambda+\beta, \varepsilon_{4}\right)}{\left(\beta, \varepsilon_{4}\right)}=\nu_{3}, \quad \frac{\left(\Lambda+\beta, \varepsilon_{1}-\varepsilon_{2}\right)}{\left(\beta, \varepsilon_{1}-\varepsilon_{2}\right)}=\frac{\nu_{2}+\nu_{3}+\nu_{4}}{3} \text {, } \\
& \frac{\left(\Lambda+\beta, \varepsilon_{1}-\varepsilon_{3}\right)}{\left(\beta, \varepsilon_{1}-\varepsilon_{3}\right)}=\frac{\nu_{1}+\nu_{2}+\nu_{3}+\nu_{4}}{4}, \quad \frac{\left(\Lambda+\beta, \varepsilon_{1}-\varepsilon_{4}\right)}{\left(\beta, \varepsilon_{1}-\varepsilon_{4}\right)}=\frac{\nu_{1}+2 \nu_{2}+\nu_{3}+\nu_{4}}{5}, \\
& \frac{\left(\Lambda+\beta, \varepsilon_{2}-\varepsilon_{3}\right)}{\left(\beta, \varepsilon_{2}-\varepsilon_{3}\right)}=\nu_{1}, \quad \frac{\left(\Lambda+\beta, \varepsilon_{2}-\varepsilon_{4}\right)}{\left(\beta, \varepsilon_{2}-\varepsilon_{4}\right)}=\frac{\nu_{1}+\nu_{2}}{2}, \quad \frac{\left(\Lambda+\beta, \varepsilon_{3}-\varepsilon_{4}\right)}{\left(\beta, \varepsilon_{3}-\varepsilon_{4}\right)}=\nu_{2} \text {, } \\
& \frac{\left(\Lambda+\beta, \varepsilon_{1}+\varepsilon_{2}\right)}{\left(\beta, \varepsilon_{1}+\varepsilon_{2}\right)}=\frac{2 \nu_{1}+3 \nu_{2}+2 \nu_{3}+\nu_{4}}{8}, \\
& \frac{\left(\Lambda+\beta, \varepsilon_{1}+\varepsilon_{3}\right)}{\left(\beta, \varepsilon_{1}+\varepsilon_{3}\right)}=\frac{\nu_{1}+3 \nu_{2}+2 \nu_{3}+\nu_{4}}{7}, \\
& \frac{\left(\Lambda+\beta, \varepsilon_{1}+\varepsilon_{4}\right)}{\left(\beta, \varepsilon_{1}+\varepsilon_{4}\right)}=\frac{\nu_{1}+2 \nu_{2}+2 \nu_{3}+\nu_{4}}{6}, \\
& \frac{\left(\Lambda+\beta, \varepsilon_{2}+\varepsilon_{3}\right)}{\left(\beta, \varepsilon_{2}+\varepsilon_{3}\right)}=\frac{\nu_{1}+2 \nu_{2}+\nu_{3}}{4}, \quad \frac{\left(\Lambda+\beta, \varepsilon_{2}+\varepsilon_{4}\right)}{\left(\beta, \varepsilon_{2}+\varepsilon_{4}\right)}=\frac{\nu_{1}+\nu_{2}+\nu_{3}}{3}, \\
& \frac{\left(\Lambda+\beta, \varepsilon_{3}+\varepsilon_{4}\right)}{\left(\beta, \varepsilon_{3}+\varepsilon_{4}\right)}=\frac{\nu_{2}+\nu_{3}}{2} \text {, } \\
& \frac{\left(\Lambda+\beta, \frac{1}{2}\left(\varepsilon_{1}+\varepsilon_{2}+\varepsilon_{3}+\varepsilon_{4}\right)\right)}{\left(\beta, \frac{1}{2}\left(\varepsilon_{1}+\varepsilon_{2}+\varepsilon_{3}+\varepsilon_{4}\right)\right)}=\frac{2 \nu_{1}+4 \nu_{2}+3 \nu_{3}+\nu_{4}}{10}, \\
& \frac{\left(\Lambda+\beta, \frac{1}{2}\left(\varepsilon_{1}+\varepsilon_{2}+\varepsilon_{3}-\varepsilon_{4}\right)\right)}{\left(\beta, \frac{1}{2}\left(\varepsilon_{1}+\varepsilon_{2}+\varepsilon_{3}-\varepsilon_{4}\right)\right)}=\frac{2 \nu_{1}+4 \nu_{2}+2 \nu_{3}+\nu_{4}}{9}, \\
& \frac{\left(\Lambda+\beta, \frac{1}{2}\left(\varepsilon_{1}+\varepsilon_{2}-\varepsilon_{3}+\varepsilon_{4}\right)\right)}{\left(\beta, \frac{1}{2}\left(\varepsilon_{1}+\varepsilon_{2}-\varepsilon_{3}+\varepsilon_{4}\right)\right)}=\frac{2 \nu_{1}+2 \nu_{2}+2 \nu_{3}+\nu_{4}}{7},
\end{aligned}
$$




$$
\begin{aligned}
& \frac{\left(\Lambda+\beta, \frac{1}{2}\left(\varepsilon_{1}+\varepsilon_{2}-\varepsilon_{3}-\varepsilon_{4}\right)\right)}{\left(\beta, \frac{1}{2}\left(\varepsilon_{1}+\varepsilon_{2}-\varepsilon_{3}-\varepsilon_{4}\right)\right)}=\frac{2 \nu_{1}+2 \nu_{2}+\nu_{3}+\nu_{4}}{6}, \\
& \frac{\left(\Lambda+\beta, \frac{1}{2}\left(\varepsilon_{1}-\varepsilon_{2}+\varepsilon_{3}+\varepsilon_{4}\right)\right)}{\left(\beta, \frac{1}{2}\left(\varepsilon_{1}-\varepsilon_{2}+\varepsilon_{3}+\varepsilon_{4}\right)\right)}=\frac{2 \nu_{2}+2 \nu_{3}+\nu_{4}}{5}, \\
& \frac{\left(\Lambda+\beta, \frac{1}{2}\left(\varepsilon_{1}-\varepsilon_{2}-\varepsilon_{3}+\varepsilon_{4}\right)\right)}{\left(\beta, \frac{1}{2}\left(\varepsilon_{1}-\varepsilon_{2}-\varepsilon_{3}+\varepsilon_{4}\right)\right)}=\frac{\nu_{3}+\nu_{4}}{2}, \\
& \frac{\left(\Lambda+\beta, \frac{1}{2}\left(\varepsilon_{1}-\varepsilon_{2}+\varepsilon_{3}-\varepsilon_{4}\right)\right)}{\left(\beta, \frac{1}{2}\left(\varepsilon_{1}-\varepsilon_{2}+\varepsilon_{3}-\varepsilon_{4}\right)\right)}=\frac{2 \nu_{2}+\nu_{3}+\nu_{4}}{4}, \\
& \frac{\left(\Lambda+\beta, \frac{1}{2}\left(\varepsilon_{1}-\varepsilon_{2}-\varepsilon_{3}-\varepsilon_{4}\right)\right)}{\left(\beta, \frac{1}{2}\left(\varepsilon_{1}-\varepsilon_{2}-\varepsilon_{3}-\varepsilon_{4}\right)\right)}=\nu_{4} .
\end{aligned}
$$

Следовательно,

$$
\begin{aligned}
& d(\Lambda+\beta)=\frac{\nu_{1} \nu_{2} \nu_{3} \nu_{4}}{2414168064 \cdot 10^{4}}\left(\nu_{1}+\nu_{2}\right)\left(\nu_{2}+\nu_{3}\right)\left(\nu_{3}+\nu_{4}\right)\left(2 \nu_{2}+\nu_{3}\right) \\
& \times\left(2 \nu_{1}+2 \nu_{2}+\nu_{3}\right)\left(\nu_{1}+2 \nu_{2}+\nu_{3}\right)\left(\nu_{1}+\nu_{2}+\nu_{3}\right)\left(\nu_{2}+\nu_{3}+\nu_{4}\right) \\
& \times\left(2 \nu_{2}+2 \nu_{3}+\nu_{4}\right)\left(2 \nu_{2}+\nu_{3}+\nu_{4}\right)\left(\nu_{1}+\nu_{2}+\nu_{3}+\nu_{4}\right) \\
& \times\left(\nu_{1}+2 \nu_{2}+\nu_{3}+\nu_{4}\right)\left(2 \nu_{1}+4 \nu_{2}+3 \nu_{3}+2 \nu_{4}\right) \\
& \times\left(2 \nu_{1}+3 \nu_{2}+2 \nu_{3}+\nu_{4}\right)\left(\nu_{1}+3 \nu_{2}+2 \nu_{3}+\nu_{4}\right) \\
& \times\left(\nu_{1}+2 \nu_{2}+2 \nu_{3}+\nu_{4}\right)\left(2 \nu_{1}+4 \nu_{2}+3 \nu_{3}+\nu_{4}\right) \\
& \times\left(2 \nu_{1}+4 \nu_{2}+2 \nu_{3}+\nu_{4}\right)\left(2 \nu_{1}+2 \nu_{2}+\nu_{3}+\nu_{4}\right) \\
& \times\left(2 \nu_{1}+2 \nu_{2}+2 \nu_{3}+\nu_{4}\right) \text {. }
\end{aligned}
$$

Замечание 3. Из (17) и (18) вытекает, что

$$
\lambda(\Lambda)=0 \Leftrightarrow \Lambda=0 \Leftrightarrow \nu_{i}=1, \quad i=1,2,3,4,
$$

и, следовательно, $\sigma\left(\lambda_{0}\right)=d^{2}(0+\beta)=1$, что соответствует формуле (1). Кроме того, на основании предложения 1 старшим весом присоединенного представления $\mathrm{Ad}$ является максимальный корень $\widetilde{\alpha}=\bar{\omega}_{1}$, для которого $\nu_{1}=2$ и $\nu_{2}=\nu_{3}=\nu_{4}=1$. Поэтому собственное значение и размерность представления Ad можно вычислить следующим образом:

$$
\begin{gathered}
\lambda_{\mathrm{Ad}}=\lambda(\widetilde{\alpha})=-\frac{1}{72 \gamma}\left(13^{2}+7^{2}+3^{2}+1^{2}-156\right)=-\frac{1}{\gamma}, \\
\operatorname{dim} \mathrm{Ad}=d(\widetilde{\alpha}+\beta)=52,
\end{gathered}
$$

что соответствует формуле (2) при $\gamma=1$ с учетом равенства $\operatorname{dim} F_{4}=52$ из табл. 1. 
5) Указанные выше простые корни и фундаментальные веса алгебры Ли $\mathfrak{f}_{4}$ задают решетки $\Lambda_{0}\left(F_{4}\right)$ и $\Lambda_{1}\left(F_{4}\right)$ соответственно следующим образом:

$$
\begin{aligned}
& \Lambda_{0}\left(F_{4}\right)=\left\{\sum_{i=1}^{4} \Psi_{i} \alpha_{i} \mid \Psi_{i} \in \mathbb{Z}, i=1, \ldots, 4\right\}, \\
& \Lambda_{1}\left(F_{4}\right)=\left\{\sum_{i=1}^{4} \Lambda_{i} \bar{\omega}_{i} \mid \Lambda_{i} \in \mathbb{Z}, i=1, \ldots, 4\right\} .
\end{aligned}
$$

После выражения корней через фундаментальные веса

$\alpha_{1}=2 \bar{\omega}_{1}-\bar{\omega}_{2}, \quad \alpha_{2}=-\bar{\omega}_{1}+2 \bar{\omega}_{2}-2 \bar{\omega}_{3}, \quad \alpha_{3}=-\bar{\omega}_{2}+2 \bar{\omega}_{3}-\bar{\omega}_{4}, \quad \alpha_{4}=-\bar{\omega}_{3}+2 \bar{\omega}_{4}$ получим

$$
\begin{aligned}
\Lambda_{0}\left(F_{4}\right)=\{ & \left(2 \Psi_{1}-\Psi_{2}\right) \bar{\omega}_{1}+\left(-\Psi_{1}+2 \Psi_{2}-\Psi_{3}\right) \bar{\omega}_{2}+\left(-2 \Psi_{2}+2 \Psi_{3}-\Psi_{4}\right) \bar{\omega}_{3} \\
& \left.+\left(-\Psi_{3}+2 \Psi_{4}\right) \bar{\omega}_{4} \mid \Psi_{i} \in \mathbb{Z}, i=1,2,3,4\right\} .
\end{aligned}
$$

Пусть $\theta: \mathbb{R}^{4} \rightarrow \mathbb{R}^{4}$ - линейное преобразование, заданное следующим образом:

$$
\left(\Psi_{1}, \Psi_{2}, \Psi_{3}, \Psi_{4}\right) \rightarrow\left(2 \Psi_{1}-\Psi_{2},-\Psi_{1}+2 \Psi_{2}-\Psi_{3},-2 \Psi_{2}+2 \Psi_{3}-\Psi_{4},-\Psi_{3}+2 \Psi_{4}\right) .
$$

Так как $\theta$ задается целочисленной матрицей с единичным определителем, то $\theta$ является изоморфизмом решетки $\mathbb{Z}^{4}$ на себя. Поэтому

$$
\Lambda_{0}\left(F_{4}\right)=\Lambda_{1}\left(F_{4}\right)
$$

решетка $\Lambda\left(F_{4}\right)$, удовлетворяющая соотношению $\Lambda_{0}\left(F_{4}\right) \subseteq \Lambda(G) \subseteq \Lambda_{1}\left(F_{4}\right)$, единственна и совпадает с $\Lambda_{1}\left(F_{4}\right)$.

Таким образом, нужно вычислить спектр только для группы $F_{4}$ с характеристической решеткой $\Lambda_{1}\left(F_{4}\right)$.

6) Из формулы (19) получаем множество старших весов группы $F_{4}$

$$
\Lambda^{+}\left(F_{4}\right)=\left\{\sum_{i=1}^{4} \Lambda_{i} \bar{\omega}_{i} \mid \Lambda_{i} \in \mathbb{Z}, \Lambda_{i} \geq 0, i=1, \ldots, 4\right\} .
$$

7) Пусть

$$
\Lambda=\sum_{i=1}^{4} \Lambda_{i} \bar{\omega}_{i} \in \Lambda^{+}\left(F_{4}\right)
$$

Тогда на основании п. 4 собственное число $\lambda(\Lambda)$ и размерность $d(\Lambda+\beta)$ вычисляются по формулам (17) и (18) соответственно, где $\nu_{i}=\Omega_{i}+1$, $\nu_{i} \in \mathbb{N}, i=1,2,3,4$. 
8) Применяя формулу (5) и полученные до этого результаты, находим кратность собственного значения $\lambda$ :

$$
\begin{aligned}
\sigma(\Lambda)=\frac{1}{24} & 4168064^{2} \cdot 10^{8} \sum_{\Xi_{12}} \nu_{1}^{2} \nu_{2}^{2} \nu_{3}^{2} \nu_{4}^{2}\left(\nu_{1}+\nu_{2}\right)^{2}\left(\nu_{2}+\nu_{3}\right)^{2}\left(2 \nu_{2}+\nu_{3}+\nu_{4}\right)^{2} \\
& \times\left(\nu_{3}+\nu_{4}\right)^{2}\left(2 \nu_{2}+\nu_{3}\right)^{2}\left(2 \nu_{1}+2 \nu_{2}+\nu_{3}\right)^{2}\left(\nu_{1}+2 \nu_{2}+\nu_{3}\right)^{2} \\
& \times\left(\nu_{1}+\nu_{2}+\nu_{3}\right)^{2}\left(\nu_{2}+\nu_{3}+\nu_{4}\right)^{2}\left(2 \nu_{2}+2 \nu_{3}+\nu_{4}\right)^{2} \\
& \times\left(\nu_{1}+\nu_{2}+\nu_{3}+\nu_{4}\right)^{2}\left(\nu_{1}+2 \nu_{2}+\nu_{3}+\nu_{4}\right)^{2} \\
& \times\left(2 \nu_{1}+4 \nu_{2}+3 \nu_{3}+2 \nu_{4}\right)^{2}\left(2 \nu_{1}+3 \nu_{2}+2 \nu_{3}+\nu_{4}\right)^{2} \\
& \times\left(\nu_{1}+3 \nu_{2}+2 \nu_{3}+\nu_{4}\right)^{2}\left(\nu_{1}+2 \nu_{2}+2 \nu_{3}+\nu_{4}\right)^{2} \\
& \times\left(2 \nu_{1}+4 \nu_{2}+3 \nu_{3}+\nu_{4}\right)^{2}\left(2 \nu_{1}+4 \nu_{2}+2 \nu_{3}+\nu_{4}\right)^{2} \\
& \times\left(2 \nu_{1}+2 \nu_{2}+\nu_{3}+\nu_{4}\right)^{2}\left(2 \nu_{1}+2 \nu_{2}+2 \nu_{3}+\nu_{4}\right)^{2}
\end{aligned}
$$

где

$$
\begin{gathered}
\Xi_{12}=\left\{\left(2 \nu_{1}+4 \nu_{2}+3 \nu_{3}+2 \nu_{4}\right)^{2}+\left(2 \nu_{1}+2 \nu_{2}+\nu_{3}\right)^{2}+\left(2 \nu_{2}+\nu_{3}\right)^{2}+\nu_{3}^{2}\right. \\
=156-72 \gamma \lambda\} .
\end{gathered}
$$

Наименьшее по модулю ненулевое значение лапласиана равно $-\frac{2}{3 \gamma}$ и соответствует неприводимому представлению группы Ли со старшим весом $\bar{\omega}_{4}$. Размерность этого представления равна 26. Следовательно, кратность собственного значения $-\frac{2}{3 \gamma}$ равна $26^{2}=676$.

\section{$\S 4$. Теоретико-числовые критерии собственных значений лапласиана}

В табл. 2-4 представлены (по убыванию) 10 наибольших отрицательных собственных значений лапласиана на группах (с биинвариантной метрикой $\nu) \mathrm{SU}(5), \operatorname{PSU}(5)$ и $F_{4}$ соответственно.

Теорема 2. Пусть $G$ - это $\mathrm{SU}(5)$ с биинвариантной римановой метрикой $\nu$ такой, что $\nu(e)=-k_{\mathrm{ad}}$. Число $\lambda<0$ является собственным значением лапласиана на $(G, \nu)$ тогда и только тогда, когда выполняются следующие условия:

1) $-50 \lambda \in \mathbb{N}$;

2) выполнено хотя бы одно из следующих условий:

2.1) натуральное число $200-200 \lambda$ можно представить в виде

$$
5 x^{2}+5 y^{2}+5 z^{2}+v^{2}, \quad \text { причем } x, y, z, v \in \mathbb{N},
$$

и

$$
\begin{gathered}
x \equiv y \equiv z(\bmod 2), \quad v \equiv x+y+z(\bmod 4) \\
x<y<z, \quad v \geq 4+x+y+z
\end{gathered}
$$




$$
\begin{array}{r}
\text { или }(\text { и) } \\
x \equiv y \equiv z(\bmod 2), \quad v+x \equiv y+z(\bmod 4), \\
x<y<z, \quad v+x \geq 4+y+z ;
\end{array}
$$

2.2) натуральное число $50-50 \lambda$ можно представить в виде

И

$$
5 x^{2}+5 y^{2}+z^{2}, \quad \text { причем } x, y, z \in \mathbb{N},
$$

$$
z \equiv x+y(\bmod 2), \quad x<y, \quad z \geq 2+x+y .
$$

При этом число старших векторов $\Lambda$ таких, что $\lambda(\Lambda)=\lambda$, равно $n_{1}+$ $n_{2}+n_{3}$, где $n_{1}\left(n_{2}\right)$ - число решений уравнения

$$
5 x^{2}+5 y^{2}+5 z^{2}+v^{2}=200-200 \lambda, \quad x, y, z, v \in \mathbb{N},
$$

удовлетворяющих условию (21) ((22) соответственно $), n_{3}$ - число решений уравнения

$$
5 x^{2}+5 y^{2}+z^{2}=50-50 \lambda, \quad x, y, z \in \mathbb{N},
$$

удовлетворяющих условию (23).

Доказательство. Следуя (13), рассмотрим диофантово уравнение

$4 \nu_{1}^{2}+6 \nu_{1} \nu_{2}+4 \nu_{1} \nu_{3}+2 \nu_{1} \nu_{4}+6 \nu_{2}^{2}+8 \nu_{2} \nu_{3}+4 \nu_{2} \nu_{4}+6 \nu_{3}^{2}+6 \nu_{3} \nu_{4}+4 \nu_{4}^{2}=50-50 \lambda$, которое можно переписать в виде

$$
\begin{aligned}
& \left(4 \nu_{1}+3 \nu_{2}+2 \nu_{3}+\nu_{4}\right)^{2}+5\left(\nu_{2}+2 \nu_{3}+\nu_{4}\right)^{2}+5\left(\nu_{2}-\nu_{4}\right)^{2}+5\left(\nu_{2}+\nu_{4}\right)^{2} \\
& \quad=200-200 \lambda
\end{aligned}
$$

где $\nu_{i} \in \mathbb{N}, i=1,2,3,4$.

Ясно, что если уравнение (26) разрешимо, то $-50 \lambda \in \mathbb{N}$.

Предположим сначала, что (26) имеет решение $\left(\nu_{1}, \nu_{2}, \nu_{3}, \nu_{4}\right) \in \mathbb{N}^{4}$ такое, что $\nu_{2}>\nu_{4}$. После замены переменных:

$$
x=\nu_{2}-\nu_{4}, \quad y=\nu_{2}+\nu_{4}, \quad z=\nu_{2}+2 \nu_{3}+\nu_{4}, \quad v=4 \nu_{1}+3 \nu_{2}+2 \nu_{3}+\nu_{4} ;
$$

уравнение (26) примет вид (24), причем будут выполнены условия (21). При этом число решений $\left(\nu_{1}, \nu_{2}, \nu_{3}, \nu_{4}\right) \in \mathbb{N}^{4}$ уравнения (26), удовлетворяющих условию $\nu_{2}>\nu_{4}$, равно числу решений уравнения $(24)$ с условием (21).

Пусть теперь уравнение (26) имеет решение $\left(\nu_{1}, \nu_{2}, \nu_{3}, \nu_{4}\right) \in \mathbb{N}^{4}$, где $\nu_{2}<\nu_{4}$. После замены переменных:

$$
x=\nu_{4}-\nu_{2}, \quad y=\nu_{2}+\nu_{4}, \quad z=\nu_{2}+2 \nu_{3}+\nu_{4}, \quad v=4 \nu_{1}+3 \nu_{2}+2 \nu_{3}+\nu_{4} ;
$$

уравнение (26) примет вид (24), причем будут выполнены условия (22). При этом число решений $\left(\nu_{1}, \nu_{2}, \nu_{3}, \nu_{4}\right) \in \mathbb{N}^{4}$ уравнения (26), удовлетворяющих условию $\nu_{2}<\nu_{4}$, равно числу решений уравнения (24) с условием (22). 
Таблица 2. Собственные значения лапласиана на $(\mathrm{SU}(5), \nu)$

\begin{tabular}{|c|c|c|c|}
\hline № & $\lambda$ & $\left(\nu_{1}, \nu_{2}, \nu_{3}, \nu_{4}\right)$ & $\sigma(\lambda)$ \\
\hline 1 & $-\frac{12}{25}$ & $\begin{array}{c}(2,1,1,1) \\
(1,1,1,2)\end{array}$ & 50 \\
\hline 2 & $-\frac{18}{25}$ & $\begin{array}{l}(1,2,1,1) \\
(1,1,2,1)\end{array}$ & 200 \\
\hline 3 & -1 & $(2,1,1,2)$ & 576 \\
\hline 4 & $-\frac{28}{25}$ & $\begin{array}{l}(3,1,1,1) \\
(1,1,1,3)\end{array}$ & 450 \\
\hline 5 & $-\frac{32}{25}$ & $\begin{array}{l}(2,1,2,1) \\
(1,2,1,2)\end{array}$ & 4050 \\
\hline 6 & $-\frac{33}{25}$ & $\begin{array}{l}(2,2,1,1) \\
(1,1,2,2)\end{array}$ & 3200 \\
\hline 7 & $-\frac{8}{5}$ & $(1,2,2,1)$ & 5625 \\
\hline 8 & $-\frac{42}{25}$ & $\begin{array}{l}(1,3,1,1) \\
(1,1,3,1)\end{array}$ & 14800 \\
\hline 9 & $-\frac{48}{25}$ & $\begin{array}{l}(2,1,1,2) \\
(2,1,1,3)\end{array}$ & \\
\hline 10 & -2 & $\begin{array}{l}(3,1,1,1,2,1,2) \\
(4,1,1,1)\end{array}$ & 63700 \\
\hline
\end{tabular}

Таблица 4. Собственные значения лапласиана на $\left(F_{4}, \nu\right)$

\begin{tabular}{|c|c|c|c|}
\hline № & $\lambda$ & $\left(\nu_{1}, \nu_{2}, \nu_{3}, \nu_{4}\right)$ & $\sigma(\lambda)$ \\
\hline 1 & $-\frac{2}{3}$ & $(1,1,1,2)$ & 676 \\
\hline 2 & -1 & $(2,1,1,1)$ & 2704 \\
\hline 3 & $-\frac{4}{3}$ & $(1,1,2,1)$ & 74529 \\
\hline 4 & $-\frac{13}{9}$ & $(1,1,1,3)$ & 104976 \\
\hline 5 & $-\frac{16}{9}$ & $(2,1,1,2)$ & 1108809 \\
\hline 6 & -2 & $(1,2,1,1)$ & 1623076 \\
\hline 7 & $-\frac{13}{6}$ & $(1,1,2,2)$ & 16777216 \\
\hline 8 & $-\frac{20}{9}$ & $(3,1,1,1)$ & 1108809 \\
\hline 9 & $-\frac{7}{3}$ & $(1,1,1,4)$ & 7033104 \\
\hline 10 & $-\frac{23}{9}$ & $(2,1,2,1)$ & 70963776 \\
\hline
\end{tabular}

Таблица 3. Собственные значения лапласиана на $(\mathrm{PSU}(5), \nu)$

\begin{tabular}{|c|c|c|c|}
\hline № & $\lambda$ & $\left(\nu_{1}, \nu_{2}, \nu_{3}, \nu_{4}\right)$ & $\sigma(\lambda)$ \\
\hline 1 & -1 & $(2,1,1,2)$ & 576 \\
\hline 2 & $-\frac{8}{5}$ & $(1,2,2,1)$ & 5625 \\
\hline 3 & -2 & $\begin{array}{l}(3,1,2,1) \\
(1,2,1,3) \\
\end{array}$ & 31752 \\
\hline 4 & $-\frac{12}{5}$ & $\begin{array}{l}(2,3,1,1) \\
(1,1,3,2) \\
(3,1,1,3)\end{array}$ & 101250 \\
\hline 5 & -3 & $\begin{array}{l}(4,2,1,1) \\
(1,1,2,4) \\
(2,2,2,2)\end{array}$ & 1148928 \\
\hline 6 & $-\frac{18}{5}$ & $\begin{array}{l}(2,1,4,1) \\
(1,4,1,2) \\
(4,1,2,2) \\
(2,2,1,4) \\
\end{array}$ & 3185000 \\
\hline 7 & -4 & $\begin{array}{l}(6,1,1,1) \\
(1,3,3,1) \\
(3,3,1,2) \\
(2,1,3,3) \\
(1,1,1,6) \\
\end{array}$ & 7201530 \\
\hline 8 & $-\frac{21}{5}$ & $(4,1,1,4)$ & 1000000 \\
\hline 9 & $-\frac{22}{5}$ & $(1,3,2,3)$ & 7290000 \\
\hline 10 & $-\frac{24}{5}$ & $\begin{array}{l}(5,2,1,2) \\
(3,2,2,3) \\
(2,1,2,5)\end{array}$ & 43640625 \\
\hline
\end{tabular}


Пусть, наконец, уравнение $(26)$ имеет решение $\left(\nu_{1}, \nu_{2}, \nu_{3}, \nu_{4}\right) \in \mathbb{N}^{4}$, где $\nu_{2}=\nu_{4}$. После замены переменных:

$$
x=\nu_{2}, \quad y=\nu_{2}+\nu_{3}, \quad z=2 \nu_{1}+2 \nu_{2}+\nu_{3} ;
$$

уравнение (26) запишется в виде (25), причем будут выполнены условия (23). При этом число решений $\left(\nu_{1}, \nu_{2}, \nu_{3}, \nu_{4}\right) \in \mathbb{N}^{4}$ уравнения $(25)$, удовлетворяющих условию $\nu_{2}=\nu_{4}$, равно числу решений уравнения $(25) \mathrm{c}$ условием (23).

Теорема 3. Пусть $G-$ это $\operatorname{PSU}(5)$ с биинвариантной римановой метрикой $\nu$ такой, что $\nu(e)=-k_{\mathrm{ad}}$. Число $\lambda<0$ является собственным значением лапласиана на $(G, \nu)$ тогда и только тогда, когда выполняются следующие условия:

1) $-10 \lambda \in \mathbb{N}$;

2) выполнено хотя бы одно из следующих условий:

2.1) натуральное число $40-40 \lambda$ можно представить в виде

$$
x^{2}+y^{2}+z^{2}+5 u^{2}, \quad \text { причем } x, y, z, u \in \mathbb{N},
$$

и

$$
\begin{gathered}
x \equiv y \equiv z \equiv u(\bmod 2), \quad u \equiv x+y+z(\bmod 4), \\
x<y<z, \quad 5 u \geq 4+x+y+z
\end{gathered}
$$

или (и)

$$
\begin{gathered}
x \equiv y \equiv z \equiv u(\bmod 2), \quad u+x+y+z \equiv 0(\bmod 4), \\
x<y<z, \quad 5 u+x \geq 4+y+z ;
\end{gathered}
$$

2.2) натуральное число $10-10 \lambda$ можно представить в виде

и

$$
x^{2}+y^{2}+5 u^{2}, \quad \text { причем } x, y, u \in \mathbb{N},
$$

$$
u \equiv x+y(\bmod 2), \quad x<y, \quad 5 u \geq 2+x+y .
$$

При этом число старших векторов $\Lambda$ таких, что $\lambda(\Lambda)=\lambda$, равно $n_{1}+$ $n_{2}+n_{3}$, где $n_{1}\left(n_{2}\right)$ - число решений уравнения

$$
x^{2}+y^{2}+z^{2}+5 u^{2}=40-40 \lambda, \quad x, y, z, u \in \mathbb{N},
$$

удовлетворяющих условиям (27) (условиям (28) соответственно), $n_{3}$ - число решений уравнения

$$
x^{2}+y^{2}+5 u^{2}=10-10 \lambda, \quad x, y, u \in \mathbb{N},
$$

удовлетворяющих условиям (29). 
Доказательство. Следуя (14), рассмотрим диофантово уравнение

$$
\begin{gathered}
4 \nu_{1}^{2}+6 \nu_{1} \nu_{2}+4 \nu_{1} \nu_{3}+2 \nu_{1} \nu_{4}+6 \nu_{2}^{2}+8 \nu_{2} \nu_{3}+4 \nu_{2} \nu_{4}+6 \nu_{3}^{2}+6 \nu_{3} \nu_{4}+4 \nu_{4}^{2} \\
=50-50 \lambda,
\end{gathered}
$$

которое можно переписать в виде (26), причем

$$
\nu_{1}+2 \nu_{2}+3 \nu_{3}+4 \nu_{4} \equiv 0(\bmod 5) .
$$

Если уравнение $(26)$ имеет решение $\left(\nu_{1}, \nu_{2}, \nu_{3}, \nu_{4}\right) \in \mathbb{N}^{4}$ такое, что $\nu_{2}>\nu_{4}$, то, как показано в доказательстве теоремы 2, его можно записать в виде (24), причем будет выполнено условие (21). При этом условие (32) будет эквивалентно условию

$$
v-5 x+5 y+5 z \equiv 0(\bmod 20) .
$$

Отсюда следует, что $v=5 u, u \in \mathbb{N}$, и $u+y+z \equiv x(\bmod 4)$; легко видеть, что последнее сравнение вместе с условием (21) образует условие (27). При этом уравнение (24) можно записать в виде $(30)$. Тогда $-40 \lambda \in \mathbb{N}$, что в совокупности с условием $-50 \lambda \in \mathbb{N}$ теоремы 2 дает условие $-10 \lambda \in \mathbb{N}$.

Если уравнение (26) имеет решение $\left(\nu_{1}, \nu_{2}, \nu_{3}, \nu_{4}\right) \in \mathbb{N}^{4}$, где $\nu_{2}<\nu_{4}$, то, как показано в доказательстве теоремы 2, его можно записать в виде (24), причем будет выполнено условие (22). При этом условие (32) будет эквивалентно условию

$$
v+5 x+5 y+5 z \equiv 0(\bmod 20) .
$$

Отсюда следует, что $v=5 u, u \in \mathbb{N}$, и

$$
u+x+y+z \equiv x(\bmod 4) ;
$$

легко видеть, что последнее сравнение вместе с условием (22) образует условие (28). При этом уравнение (24) можно записать в виде (30). Тогда $-40 \lambda \in \mathbb{N}$, что в совокупности с условием $-50 \lambda \in \mathbb{N}$ теоремы 2 дает условие $-10 \lambda \in \mathbb{N}$.

Если уравнение (26) имеет решение $\left(\nu_{1}, \nu_{2}, \nu_{3}, \nu_{4}\right) \in \mathbb{N}^{4}$, где $\nu_{2}=\nu_{4}$, то, как показано в доказательстве теоремы 2, его можно записать в виде (25), причем будет выполнено условие (23). При этом условие (32) будет эквивалентно условию

$$
z+5 x+5 y \equiv 0(\bmod 20)
$$

Отсюда следует, что $z=5 u, u \in \mathbb{N}$, и

$$
u+x+y \equiv 0(\bmod 2)
$$

последнее равенство вместе с условием (23) образует условие (29). При этом уравнение $(25)$ можно записать в виде $(31)$, и $-10 \lambda \in \mathbb{N}$. 
Теорема 4. Пусть $G-$ это $F_{4}$ с биинвариантной римановой метрикой $\nu$ такой, что $\nu(e)=-k_{\mathrm{ad}}$. Число $\lambda<0$ является собственным значением лапласиана на $(G, \nu)$ тогда и только тогда, когда выполняются следующие условия:

1) $-72 \lambda \in \mathbb{N}$

2) натуральное число $156-72 \lambda$ можно представить в виде

$$
x^{2}+y^{2}+z^{2}+v^{2}, \quad x, y, z, v \in \mathbb{N},
$$

причем

$$
x \equiv y \equiv z \equiv v(\bmod 2), \quad x<y<z, \quad v \geq 2+x+y+z .
$$

При этом число старших векторов $\Lambda$ таких, что $\lambda(\Lambda)=\lambda$, равно количеству таких представлений, удовлетворяющих условию (33).

Доказательство. Следуя (20), рассмотрим диофантово уравнение

$$
\left(2 \nu_{1}+4 \nu_{2}+3 \nu_{3}+2 \nu_{4}\right)^{2}+\left(2 \nu_{1}+2 \nu_{2}+\nu_{3}\right)^{2}+\left(2 \nu_{2}+\nu_{3}\right)^{2}+\nu_{3}^{2}=156-72 \lambda,
$$

где $\nu_{1}, \nu_{2}, \nu_{3}, \nu_{4} \in \mathbb{N}$.

Ясно, что если уравнение (34) разрешимо, то $-72 \lambda \in \mathbb{N}$. Если ввести следующие обозначения:

$$
x=\nu_{3}, \quad y=2 \nu_{2}+\nu_{3}, \quad z=2 \nu_{1}+2 \nu_{2}+\nu_{3}, \quad v=2 \nu_{1}+4 \nu_{2}+3 \nu_{3}+2 \nu_{4} ;
$$

то уравнение (34) запишется в виде $x^{2}+y^{2}+z^{2}+v^{2}=156-72 \lambda$, причем будут выполнены условия (33).

Автор благодарна профессору В. Н. Берестовскому за постановку задачи и полезные замечания.

\section{Список литературы}

1. Берестовский В. Н., Зубарева И. А., Свиркин В. М. Спектр оператора Лапласа на связных компактных простых группах Ли ранга три // Матем. тр. 2016. Т. 19, № 1. С. 3-45.

2. Берестовский В. Н., Свиркин В. М. Оператор Лапласа на однородных нормальных римановых многообразиях // Матем. тр. 2009. Т. 12, № 2 . C. 3-40.

3. Берестовский В. Н., Свиркин В. М. Спектр оператора Лапласа на компактных односвязных простых группах Ли ранга два // Физикоматематические науки / Учен. зап. Казан. гос. ун-та. Сер. Физ.матем. науки. 2009. Т. 151, № 4. С. 15-35. 
4. Бурбаки Н. Группы и алгебры Ли. Гл. IV-VI. М.: Мир, 1972.

5. Зубарева И. А. Спектр оператора Лапласа на некоторых связных компактных простых группах Ли ранга четыре // Матем. тр. 2016. Т. 19, № 2. C. $42-85$.

6. Свиркин В. М. Спектр оператора Лапласа связных компактных простых групп Ли ранга один и два // Физико-математические науки / Учен. зап. Казан. гос. ун-та. Сер. Физ.-матем. науки. 2010. Т. 152, № 1. C. 219-234.

Зубарева Ирина Александровна Институт математики им. С. Л. Соболева СО РАН, Омский филиал, ул. Певцова, 13, Омск, 644099 РОССИЯ.

E-mail: i_gribanova@mail.ru
Поступила в редакцию 10 сентября 2018 г.

Получена после доработки 10 сентября 2018 г.

Принята к публикации 27 февраля 2019 г. 